\title{
Innovative 3D Spine Form Analysis and Parametrization of Scoliosis, Lordosis, Kyphosis and Malposition with TERGOSKOP
}

\author{
Lothar PAUL ${ }^{\mathrm{a} 1}$, Heiko TOBER ${ }^{\mathrm{b} 2}$, Günther HEGEWALD ${ }^{\mathrm{b3}}$ \\ ${ }^{a}$ Gesellschaft zur Förderung angewandter Informatik e.V. Berlin (GFal), Berlin, Germany; \\ ${ }^{\mathrm{b}}$ T\&T medilogic GmbH Berlin, Germany
}

\begin{abstract}
Backache, spine deformations and physiologic malpositions are known as widespread negative phenomena in our modern world. Analysis of the outer shape and back surface of a single patient one of the basic elements in the chain of reasoning and evaluation - is usually performed by orthopedic specialists which may decide about treatment and the use of correction implements. We present an innovative solution for 3D back measurement and scientific evaluation, designed to simplify and objectify spine curvature in connection with the position of the body's gravity centre (COG). The solution TERGOSKOP represents a tool for diagnostic investigation, proof of rehabilitation progress and inter-case comparison of spine curvature characteristics.
\end{abstract}

Keywords: body measures, spine, orthopedics, backache, body parameters, deformation, 3D measurement, TERGOSKOP, COG

\section{Introduction}

\subsection{Motivation and background}

About $40 \%$ of the whole German population (all ages) often or permanently fight with painful backache and due to estimates, between 60 and $80 \%$ do have at least a one-time sad experience with it. About 5 to $10 \%$ of all sick certificates in Germany are related to spinal and back disorders. This numbers give a vivid illustration of the enormous economic expenses standing behind the issue. And even more because enduring spinal disorders usually lead to lasting disability and in many cases even occupational or permanent invalidity.

Primarily, the number of back disorders can probably be seen as an implication of changes in lifestyle and particularly the working environment (preliminary sedentary activity, akinesia, maladjusted nutrition etc.). Another not negligible portion represent medical conditions related to more or less complex and serious three-dimensional deformities of the spine, the actual causes of which are not finally ascertained. It is particularly documented, that genes play a role in their formation [3], but in many cases no explicit origin is diagnosable and generally the causes are believed to be multifactorial or, if the condition emerges during biological growth - idiopathic. In particular cases, pathologic spine form deviations are identified as results of degenerative diseases (e.g. arthritis), developmental problems, osteoporosis / compression fractures or trauma [4]. Though a much larger number of particular syndromes, classifications and designations is in use, most commonly these physiological conditions are known as scoliosis, lordosis, and kyphosis (fig1).

The range of possible pathological implications of the named phenomena varies. In some incidents the problem resolves on it's own, e.g. as a concerned child grows. In other incidents, entailed implications range from cosmetical challenges and casual pain to serious damage of spine, chest, pelvis, heart or lung. In heavy-grade cases and if left untreated, significant constraints may threaten in quality and even expectation of life.

paul@gfai.de ; www.gfai.de/forschungsbereiche/3d/3ddv/

tober@medilogic.com ; www.medilogic.com

hegewald@medilogic.com ; www.medilogic.com 

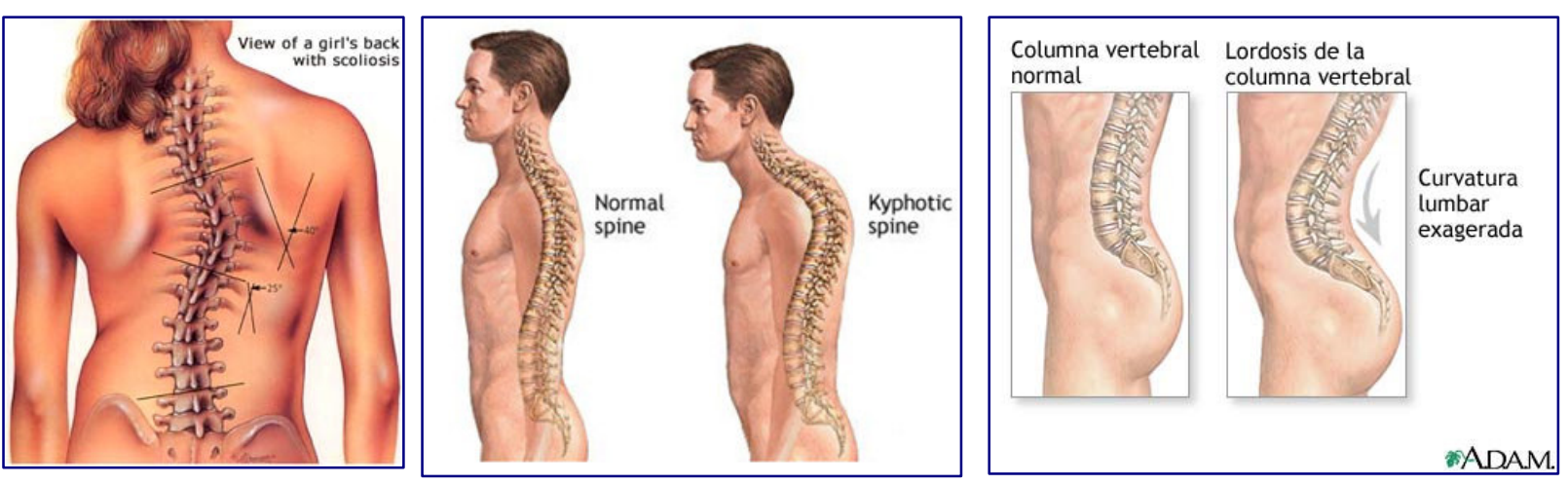

Fig. 1: Illustrations of most widespread pathological spine form deformations scoliosis, kyphosis and lordosis (images from [6][7][10]))

The prevalence of problematic spine deformation cases is different for the individual medical condition types which are unequally distributed over regions, genders and ages. In fact, a remarkable scoliosis diagnosis $\left(>10^{\circ} \mathrm{Cobb}\right)$ is consistently ascertained for about $1 \%$ of the population worldwide (here females are affected four times more than males). That means, this condition on its own concerns many million people.

\subsection{Measurement of spine deformities in diagnosis and treatment}

In nearly all cases the initial diagnosis of a spine deformation as scoliosis and kyphosis is made from evaluations of the outward individual appearance. For detailed diagnosis, prognosis and medical decisions, of course, X-ray and tomographic investigations represent the method of choice. However, the aspect of cumulative radiation exposure may become particularly problematic regarding frequent monitoring of juvenile patients.

Goniometers are the most established and simple devices for spine curvature measurement. They represent a quick and cost effective method to classify and quantify a patients bearing and the backbone mobility by measuring different characteristic angles. Such angles, for example, are those between backbone and a vertical drawn from the seventh cervical, and the angle describing the lumbosacral transition (transition from lumbar spine to sacral region

Scoliometers are mechanical or electronic devices (slope-finders, levels) which are used to estimate the amount of characteristic for skoliosis asymmetry in a person's torso. Scoliometers are widely used in school screenings, the determined scoliometer values play an important role in clinical follow-up checks.

However, for comprehensive bearing diagnosis these simple measurement methods are suitable to a limited extend only.

Today, contactless optical scanning methods offer promising possibilities for objective and comparable form data acquisition, which can serve for individual diagnosis and treatment tracking as well as for inter-case data bases and analysis. Particularly white light based (non-laser) approaches are easy to establish in body-related applications for their zero-risk category and short measurement time. Nevertheless, benefit from the thousand-fold gain in measured coordinate numbers can be achieved solely with sophisticated processing, recognition and parametrization software.

The combined measurement and data processing of both feet pressure distribution and spine surface geometry (back muscles and backbone) by means of TERGOSKOP, which is introduced in the following, provides a new efficient tool for the orthopedic applications described below. 


\section{Introduction: TERGOSKOP}

\subsection{Targeted applications}

The TERGOSKOP device was designed and is currently applicable for categorization and quantified analysis, follow-up and relapse checks of scoliosis patients. One of the motivating aspect was to minimize radiation exposure unavoidably connected with X-ray analysis.

Further applications were investigated and proved in the field of measurement, analysis and follow-up for kyphosis and lordosis cases. Diagnosis of pelvic and scapula obliquity is another available option. The system further enables detection and evaluation of leg length differences.

TERGOSKOP is particularly an appropriate solution to measure and evaluate effectiveness in therapies with orthopedic supplies and appliances. A special advantage of the solution is its combination of spine measurement with center of gravity analysis, making it a capable tool for screening measures in child-healthcare.

Generally, for comprehensive static torso analysis, the following information has to be established:

- position of torso compared to the bodies COG,

- occurrence, degree and relation of kyphosis and lordosis

- position of turning point

- angle of pelvic inclination

- lateral inclinations and torsions.

The TERGOSKOP system is well-applicable for evaluation of muscular dysfunctions and for effectiveness analysis of correcting measures and supplies. In physiotherapy, target-oriented training recommendations for the reduction of muscular dysfunctions can be derived and improved from repeated measurements.

\subsection{3d measurement method and features}

The TERGOSKOP solution uses an active, stereo-image-based and high-density topometrical measurement approach. The method is derived from the Gray Code + Phase-Shift (GC+PS) technique and combines it with active stereometry (AST) methods, the both are well described in literature [8][9]. Advantages of both methods result in high resolution, accuracy and a comparably short measurement time as well as straightforward data processing and high reliability.

As known from the combined GC+PC method, a sequence of patterns is projected onto the surface to be measured in order to accomplish quick high-density $3 \mathrm{~d}$ measurement. The sequence contains 21 different patterns and consists of the narrowing binary stripe part (Gray Code) and a set of phaseshifted grey-value sinus periods. Images of the projected and deformed pattern are captured synchronously by both cameras. Standard image data processing (pre-filtering and binarization for Gray Coded images) is applied to the images first.

Differently from GC+PC method, the subsequent data processing targeting to depth calculation does not include or use any information about the projecting unit, but relies on the captured stereo image data only, this way rather following AST methodology. In other words, the projected pattern sequence is used solely to provide a very dense grid of marks at the surface to be measured. Hence, projector matrix and the projector's course of beam is excluded from depth calculation. This way, any possible lens distortion error influences on the result from the projection side are excluded, too.

Stereo image processing usually presupposes a reliable referencing between left and right image regions or even pixels. In many cases quite complex rectification or recognition procedures are involved to achieve a pixel-wise disparity map for depth calculations. If applied not to single landmarks, but to the whole image content, such approaches are usually characterized by low performance. 
In TERGOSKOP measurement, reliable and pixel-wise stereo references are determined from the image sequence absolute-phase diagrams, using epipolar geometry algorithmic functions [1] [2]. As a result of algorithmic optimization of the depth calculation, the time our implementation takes to perform a spine measurement, is only marginal more compared to the straight forward GC+PS triangulation.

\subsection{Hardware and system layout}

The system includes two industrial 1.3 megapixel monochrome cameras arranged vertically at a basic distance and a fairly bright (2200 ANSI lumen) programmable projecting unit providing approximately the same resolution mounted in between the image sensors.

For both image sensors (cameras + lenses) internal calibration is necessary. Parameters of the unavoidable lens distortion functions are determined by means of a calibration plate with equidistant optical marks and specified in each device initialization file. Hence, the camera distortion is automatically corrected during image preprocessing procedures.

For on-site calibration and maintenance, an application-specific calibration rack was designed, which provides 75 optical marks on four different depth levels (fig. 2).

With TERGOSKOP, a lateral measurement field of 80 to 90 centimeters is covered at a measurement distance of two meters. Special efforts for environment light exclusion (black-out) are not required in room conditions due to the powerful projecting unit. However, direct sunlight or powerful illuminations in the measurement area would of course inflict losses in accuracy and reliability.

The time for a single 3D spine measurement, providing primarily up to 1.xxx million $3 \mathrm{~d}$ coordinates with assigned grey values is typically in the range of 1.2 to 1,6 seconds.
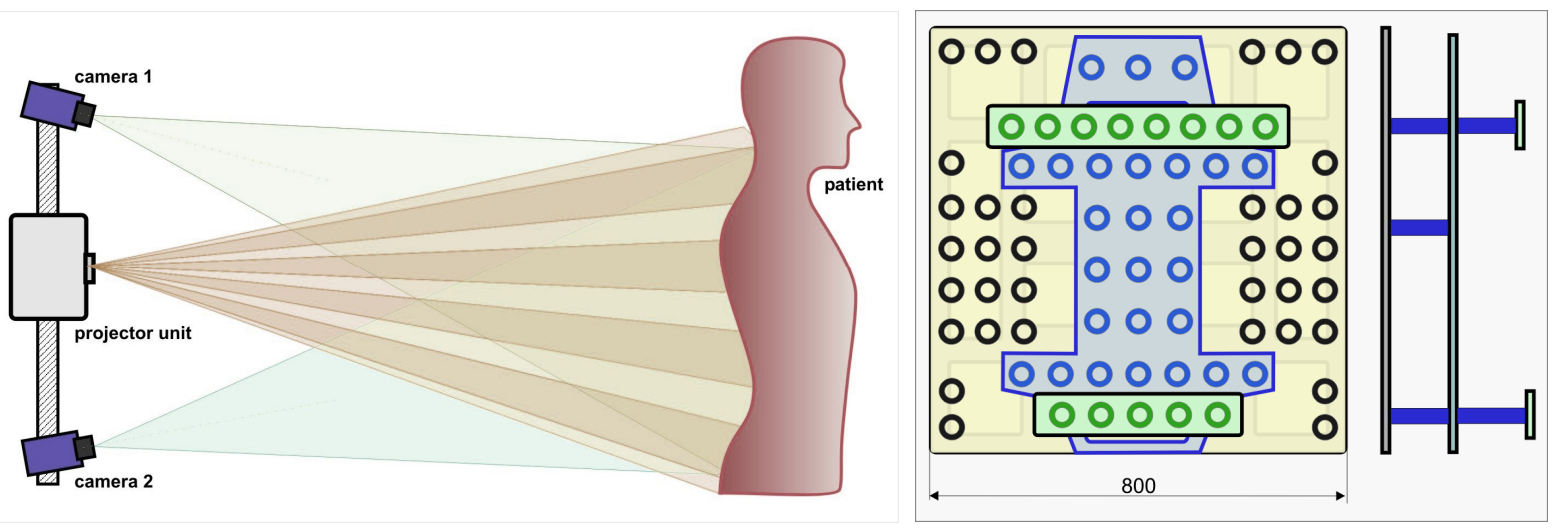

Fig. 2: Module setup (left) and calibration rack for external sensor calibration (right)

Besides 3D back surface measurement, a pressure measuring platform beneath the patient's feet is an integrated part of the solution. The combination of $3 D$ surface and $C O G$ analysis is currently considered a unique feature of TERGOSKOP systems.

The main component of the medilogic pressure measurement module is a sensor array designed as a square platform, containing 4.096 pressure sensors with a resolution of 2 Sensors $/ \mathrm{cm}^{2}$. The measured pressure data is transferred to the control PC by means of a wireless transfer modem, providing the basis for calculation of the body's COG. Coordinate systems of the 3D sensor and pressure measurement module have to be merged and calibrated in the phase of system assembly and implementation. 


\subsection{Measurement procedure and data processing}

TERGOSKOP in its current version represents a combined whole surface and marker based measurement system. The markers are solely application related, i.e. they are not necessary for 3D data acquisition, but used to specify points of interest inside the resulting $3 \mathrm{~d}$ point clouds.

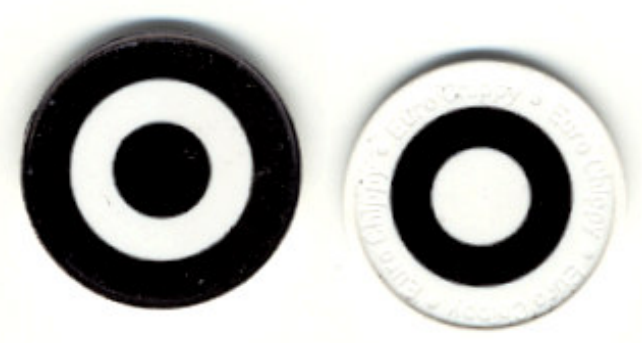

Fig. 3: Two types of flat ring-shaped markers are used for TERGOSKOP analysis

Currently, two types of circular and flat markers are used, where the second type represents the negation of the first in optical aspects (fig. 3). The mark diameter is 24 millimeters. The surface of the applied markers is measured together with and in the same procedure as the body surface. Automatic, image based marker detection is performed in the captured images. The detection of markers and the determination of the type represent truly image based algorithms not using information about the applied marker distribution, while their identification and assignment is solved using an a-priori available hypothesis about the expected regions of certain marker locations. After recognition, only the center coordinate $(\mathrm{x}, \mathrm{y}, \mathrm{z})$ and the index of a mark is considered in further processing and analysis. For accurate calculation of the center coordinates, an iterative ellipse fit algorithm is used.

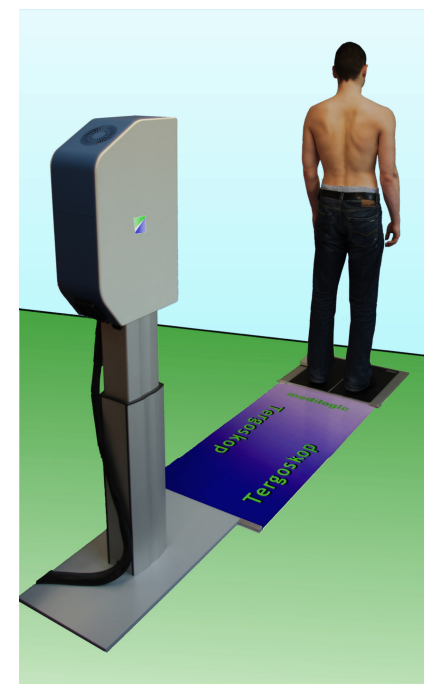

left: devices and set-up with a patient middle: typical marker positions (3D point cloud with assigned grey-value information) right: provided back and side view (detected marker positions, COG)

A medically skilled operator positions a set of marks (usually 10) on the back of the person to be measured. For backbone localization, backbone markers are placed at the following positions:

- Vertebra Prominens (C7)

- Angular point lumbar lordosis (SL)

- Sacrum point (SP) 
Between marker positions PC7 and PSL a line is drawn alongside the backbone and divided into four even sections. This way, another three marker positions are defined (PW1, PW2 and PW3). Backbone markers are shown in fig. 4 as red small circles. For pelvis and shoulder analysis the following markers are added to the scene (blue circles in fig. 4 , right):

\section{- Lumbar dimples \\ - Bladebones}

In parallel to the optical measuring of the spine, our proposed system determines the pressure distribution and calculates the centr of pressure (COP) by using a pressure measuring platform. Diagnostic investigation of abnormal postures is facilitated remarkably by the combined presentation of lines of gravity as shown in fig. 4,7 .

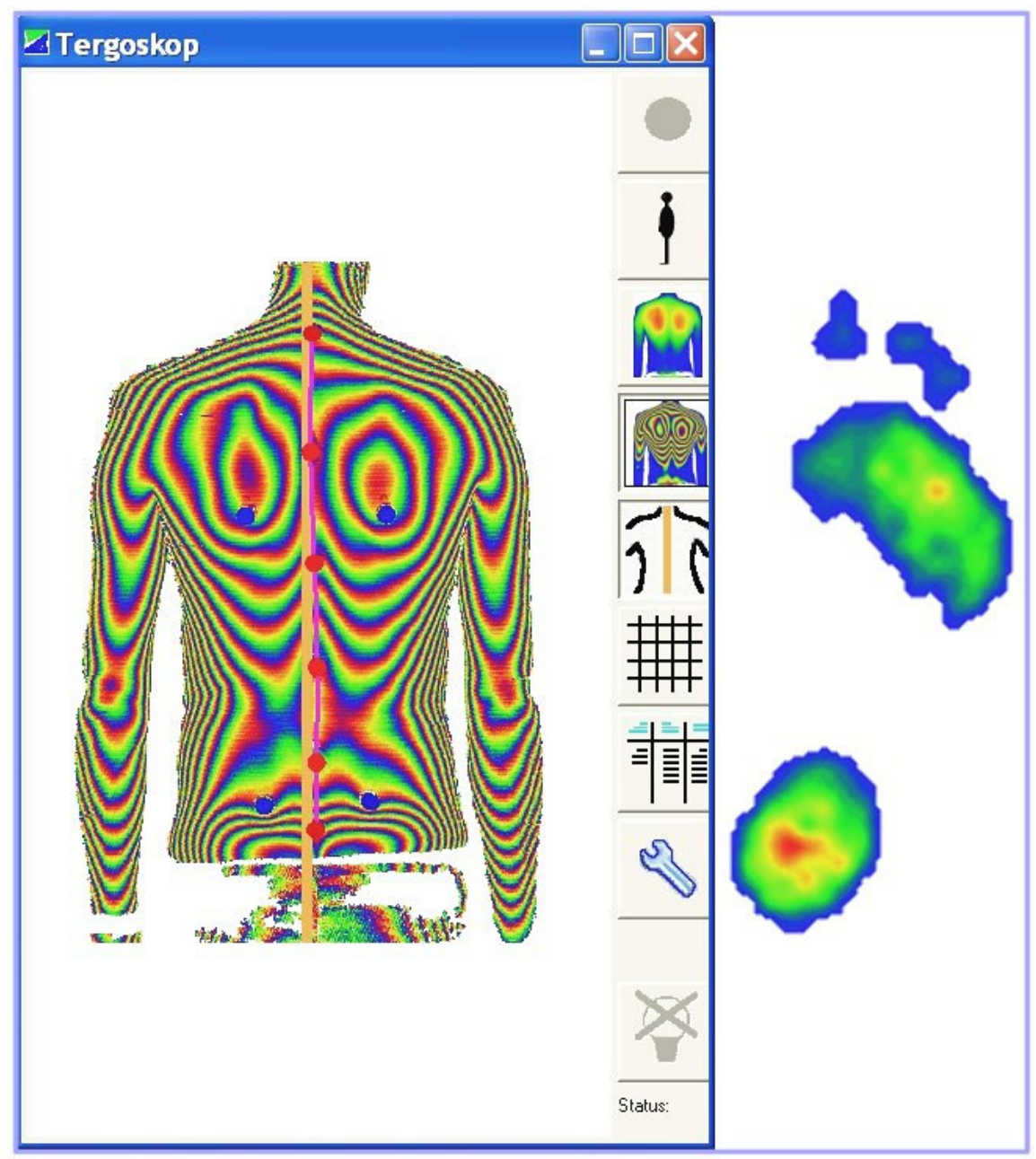

fig. 5: Screenshot: staged Tergoskop with pressure distribution (both feet are measured, only the right one is displayed here)

\section{Measurement results and calculated parameters}

For graphic assistance, the software provides a false color presentation of the three-dimensional surface of the back. Hereby, the depth information is color-coded. Beside the pressure distribution of a right foot on the right, figure 5 shows a staged Tergoskop. The perpendicular line from COP is given as an orange line.

By means of the digitized marker positions of the spinal column and the coordinates of the surface between them, a mathematical model of the backbone in the sagittal and the frontal plane can be reconstructed (fig. 7). 
Furthermore, a parametrization of the $3 d$ curve enables the analysis of curve progression. Kyphosis, lordosis and scoliosis angles are automatically calculated. Curvature indexes and planes are used to quantify the geometry of the spinal column.

Further parameters can be obtained from the evaluation of the markers at the shoulders and the pelvis. In order to parametrize the COP, it is related to the angular point of lumbar lordosis.
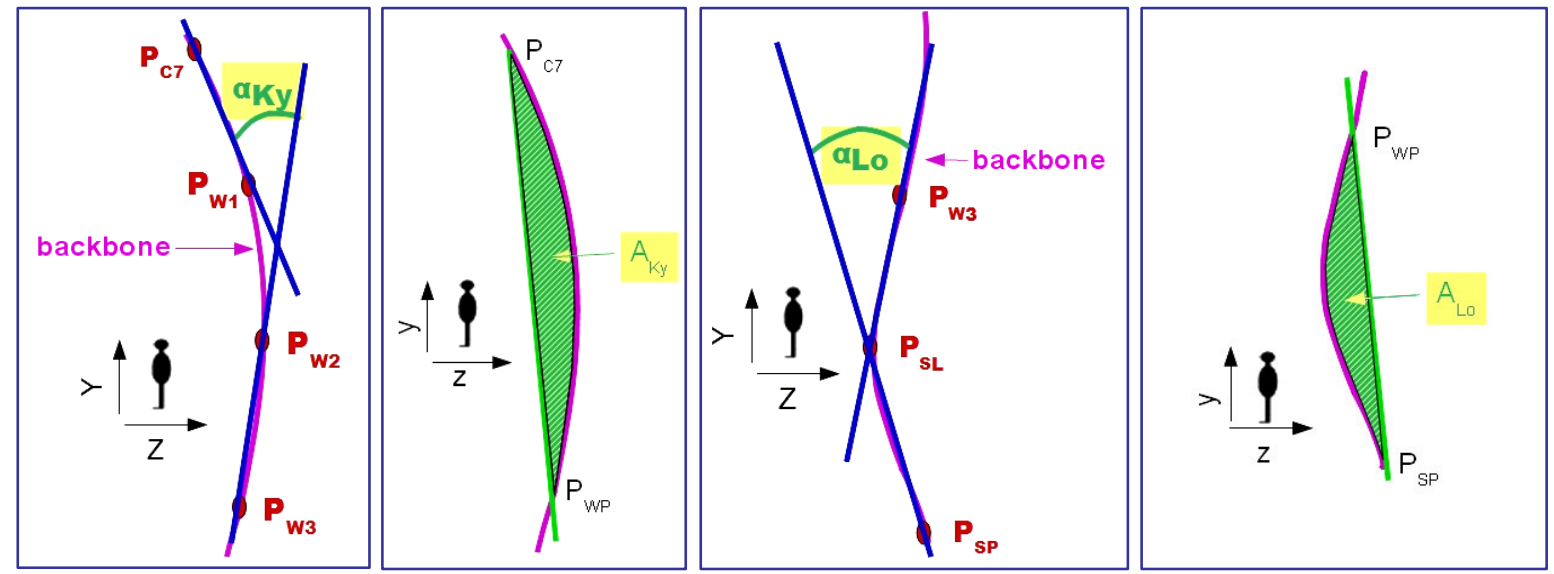

Fig. 6: Illustration of spine parameter calculation (examples)

from left to right: $a$ - kyphosic angle, $b$-kyphosic area, $c$-lordosic angle, $d$-lordosic area

To support the classification of the ongoing measuring, the currently processed dataset can be displayed and automatically compared to the average values of a selected reference group. Hereby, the challenge of classification is facilitated enormously.

Parameter calculation is performed by established and acknowledged algorithms and formulas. As an example, we present the method for kyphosis angle $\alpha_{K y}$ and lordosis angle $\alpha_{L o}$, which are calculated by the method of Klee [5] and the associated areas of spinal column curvature:

The position of spinal column markers in the sagittal plane is shown in fig. XXX. The following intermediate values are calculated:

$$
\begin{aligned}
& \alpha_{1}=\angle\left(P_{C 7}, P_{W 1}\right) \\
& \alpha_{3}=\angle\left(P_{W 2}, P_{W 3}\right) \\
& \alpha_{4}=\angle\left(P_{W 3}, P_{S L}\right) \\
& \alpha_{5}=\angle\left(P_{S L}, P_{S P}\right)
\end{aligned}
$$

From here, the kyphosis angle results in

$$
\alpha_{K y}=\alpha_{3}-\alpha_{1}
$$

and the lordosis angle in

$$
\alpha_{L o}=\alpha_{4}-\alpha_{5}
$$

Spinal column curvature areas are calculated as follows:

If $L_{R U}$ is assumed to be the length of torso, $F(y)$ - sagittal spinal column curvature, $g(y)$ - frontal spinal column curvature and $P_{W P}-$ inflexion point (sagittal plane) of spinal column curvature, then the standardized kyphosis curvature area is calculated as: 


$$
A_{K y}=\frac{\left(\int_{P_{C 7}}^{P_{W P}}|f(y)| d y\right)}{L_{R U}}\left[\mathrm{~mm}^{2} / \mathrm{mm}\right]_{\text {(fig. 6b) }}
$$

The standardized lordosis curvature area is then:

$$
A_{L o}=\frac{\left(\int_{P_{W P}}^{P_{S P}}|f(y)| d y\right)}{L_{R U}}\left[\mathrm{~mm}^{2} / \mathrm{mm}\right]_{(\text {fig. } 6 \mathrm{~d})}
$$

\section{TORSO / PELVIS}

$L_{R U} \quad$ torso length [mm]

$\alpha_{R U} \quad$ torso obliquity [ $\left.{ }^{\circ}\right]$

$\beta_{S U} \quad$ shoulder obliquity [ $\left.{ }^{0}\right]$

$\beta_{B E} \quad$ pelvic obliquity $\left[{ }^{\circ}\right]$

$\alpha_{K B} \quad$ sacroiliac declination [ [ $]$

$\lambda_{S B T} \quad$ shoulder-pelvis torsion $\left[{ }^{\circ}\right]$

\section{SPINAL COLUMN}

$I_{W} \quad$ spinal curvature index [\%]

$A_{W} \quad$ spinal curvature area $\left[\mathrm{mm}^{2} / \mathrm{mm}\right]$

\section{RELATIVE COP}

$X_{D S P} \quad \mathrm{X}$ coordinate $[\mathrm{mm}]$

$Y_{D S P} \quad \mathrm{Y}$ coordinate $[\mathrm{mm}]$

\section{KYPHOSIS}

$\alpha_{K y} \quad$ kyphotic angle [ $\left.{ }^{\circ}\right]$

$I_{K y} \quad$ kyphotic curvature index [\%]

$A_{K y} \quad$ kyphotic curvature area $\left[\mathrm{mm}^{2} / \mathrm{mm}\right]$

$S_{K y} \quad$ kyphotic chord [\%]

\section{$\underline{\text { LORDOSIS }}$}

$\alpha_{L o} \quad$ lordotic angle [ $\left.{ }^{\circ}\right]$

$I_{L o} \quad$ lordotic curvature index [\%]

$A_{\text {Lo }} \quad$ lordotic curvature area $\left[\mathrm{mm}^{2} / \mathrm{mm}\right]$

$S_{L o} \quad$ lordotic chord [\%]

\begin{tabular}{|l|c|}
\hline & SCOLIOSIS \\
\hline$I_{S k}$ & scoliotic curvature index [\%] \\
\hline$A_{S k}$ & scoliotic curvature area $\left[\mathrm{mm}^{2} / \mathrm{mm}\right]$ \\
\hline$X_{S k, l}$ & maximum left abberation [mm] \\
\hline$X_{S k, r}$ & maximum right abberation [mm] \\
\hline
\end{tabular}

Table 1: List of TERGOSKOP spine parameters

Altogether, the standard TERGOSKOP parameter list (screenshot Fig. XXX) summarises the 23 parameters presented in table 1.

\section{Conclusions and outlook}

In the present paper we introduced the device and software of our measurement solution TERGOSKOP, the first version of which was presented to the public in April 2009 at the EXPOLIFE fair in Kassel and is now in a practical test stage. 


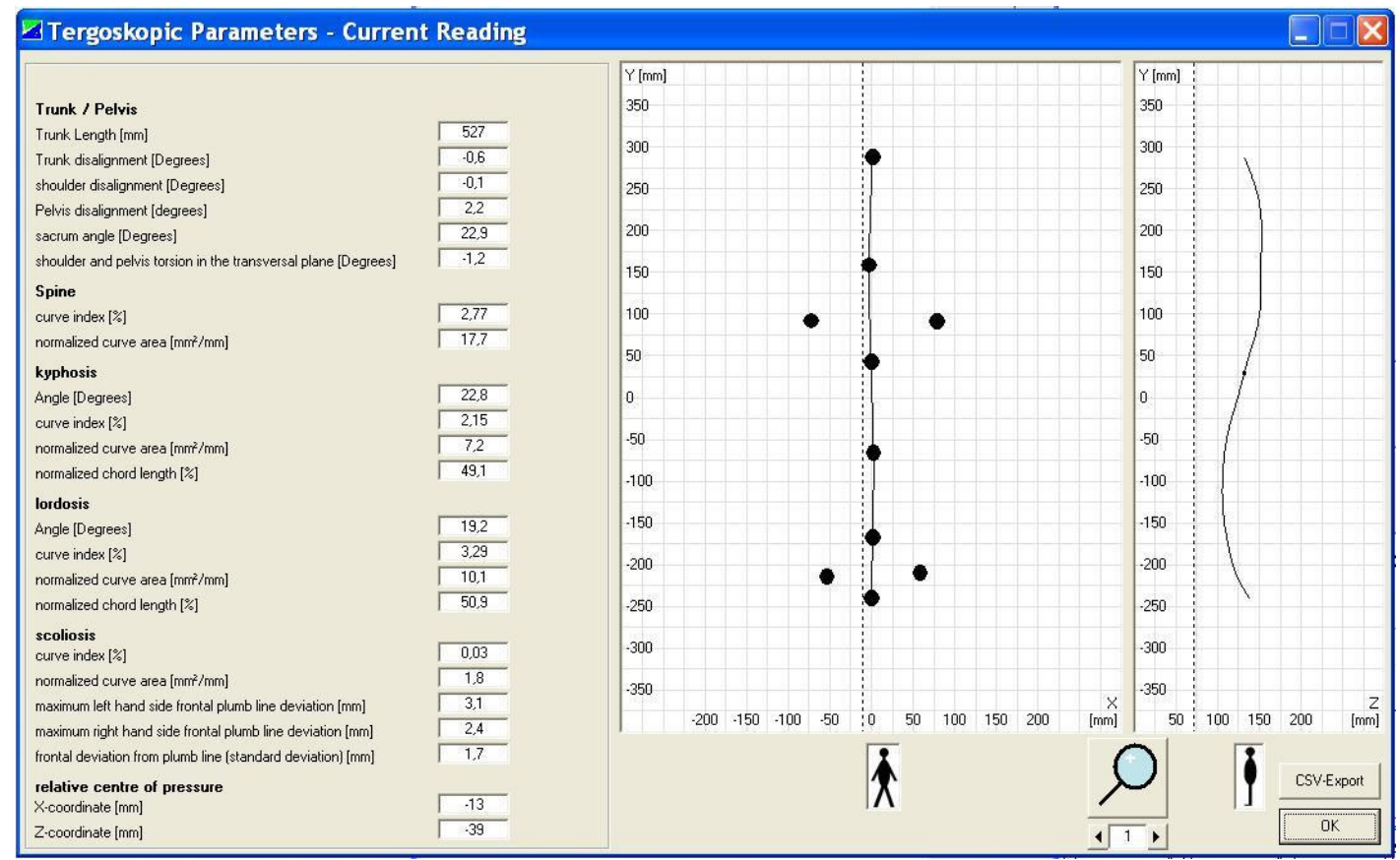

Fig. 7: Screenshot TERGOSKOP user interface: calculated parameters, measured spine

TERGOSKOP enables investigators to do precise posture analysis, without any kind of mechanical repercussion on the patient by using a fast, optical, three-dimensional measurement method. Accordingly, the system constitutes an important aid for diagnostic, treatment and follow-up examination of spine column deformity. Furthermore, by using a projector and cameras operating in normal light spectrum for the acquisition process, harmless deployment of the device is given in common orthopedic applications as well as in clinical daily routine.

Important information on body posture can be gathered when anatomical checkpoints are measured precisely by using marks and these are further related to the COP. This information includes the extent of scoliosis, kyphosis and lordosis as well as asymmetry of shoulder and pelvic region.

In continued practical tests initially several dozen male and female probands were measured in order to gather statistical data. We plan to perform an inquiry targeting at comparison of the presented here marker based measurement with markerless methods based on local 3D curvature analysis.

\section{References}

[1] Zhengyou Zhang: Determining the Epipolar Geometry and its Uncertainty: A Review. International Journal of Computer Vision, 27(2). 161-198 (1998). Kluwer Academic Publishers, Boston 1998

[2] Xu, G., Zhang Z.: Epipolar Geometry in Stereo. Motion and Object Recognition: A Unified Approach. Kluwer Academic Publishers, Boston 1996

[3] Ogilvie JW, Braun J, Argyle V, Nelson L, Meade M, Ward K: The search for idiopathic scoliosis genes. Axial Biotech, Inc., Salt Lake City, UT, USA. jogilvie@axialbiotech.com, Spine (Phila Pa 1976). 2006 Mar 15;31(6):679-81.

[4] Kouwenhoven JW, Castelein RM: The pathogenesis of adolescent idiopathic scoliosis: review of the literature, Department of Orthopedics, University Medical Center Utrecht, Utrecht, The Netherlands, Spine (Phila Pa 1976). 2008 Dec 15;33(26):2898-908.

[5] Klee A.: Haltung, muskuläre Balance und Training. Beiträge zur Sportwissenschaft Bd. 20, Herausg. R. Daugs, M. Fikus, G. Gebauer, D. Hackfort, Verlag Harry Deutsch 1994, ISBN 3-8171-1354-4

[6] Iordosis: Maryland General Hospital: http://health.marylandgeneral.org/imagepages/9583.htm

[7] kyphosis: http://chinesemedicinenews.com/wp-content/uploads/kyphosis.jpg

[8] Breuckmann, B., 1993. Bildverarbeitung und optische Messtechnik in der industriellen Praxis. Franzis, München

[9] Sasse R.: Bestimmung von Entfernungsbildern durch aktive stereoskopische Verfahren, Dissertation TU Berlin, Fortschritte der Robotik Nr. 23, Vieweg Verlag 1994

[10] scoliosis: Discover Chiropractic: http://mankatochiropractor.com/news/what-is-scoliosis/ 